\section{PSICOLOGIA IBEROAMERICANA}

\section{Psicología lberoamericana}

ISSN: 1405-0943

psicología.iberoamericana@uia.mx

Universidad Iberoamericana, Ciudad de

México

México

Toribio Pérez, Lorena; González Arratia López Fuentes, Norma Ivonne; Valdez Medina, José Luis;

González Escobar, Sergio; Van Barneveld, Hans Oudhof

Validación de la Escala de Felicidad de Alarcón para adolescentes mexicanos

Psicología Iberoamericana, vol. 20, núm. 1, enero-junio, 2012, pp. 71-79

Universidad Iberoamericana, Ciudad de México

Distrito Federal, México

Disponible en: http://www.redalyc.org/articulo.oa?id=133924623008

Cómo citar el artículo

- Número completo

- Más información del artículo

- Página de la revista en redalyc.org

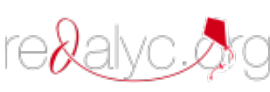

Sistema de Información Científica

Red de Revistas Científicas de América Latina, el Caribe, España y Portugal

Proyecto académico sin fines de lucro, desarrollado bajo la iniciativa de acceso abierto 


\title{
Validación de la Escala de Felicidad de Alarcón para adolescentes mexicanos
}

\author{
Validation of Alarcon's Happiness Scale in Mexican Adolescent
}

\author{
Lorena Toribio Pérez \\ Norma Ivonne González Arratia López Fuentes \\ José Luis Valdez Medina \\ Sergio González Escobar \\ Hans Oudhof Van Barneveld* \\ Facultad de Ciencias de la Conducta \\ Universidad Autónoma del Estado de México, Toluca, México
}

\section{RESUMEN}

El presente artículo tiene por objetivo obtener indicadores de validez de la Escala de Felicidad de Alarcón (2006) para adolescentes mexicanos. Se aplicó a 405 estudiantes de preparatoria, mujeres y hombres, de 14 a 19 años. La escala original comprende correlaciones ítem-escala, con coeficientes $r$ producto momento altamente significativas y un alfa total de .916. Está compuesta por 27 ítems de tipo Likert con cinco alternativas de respuesta. La validez en este estudio se estableció por análisis factorial exploratorio con el método de componentes principales y rotación ortogonal de tipo Varimax, previo al cálculo de la prueba de Kaiser-Meyer. Se obtuvieron cuatro factores que explican el 50.32\% de varianza total: sentido positivo de la vida, satisfacción con la vida, realización personal y alegría de vivir. La escala total presenta un valor satisfactorio con respecto a la puntuación total; los niveles se interpretan como un alto nivel de felicidad. En conclusión se puede considerar un instrumento con propiedades psicométricas adecuadas para la medición de la felicidad en estas edades.

Descriptores: felicidad, adolescentes, validación, satisfacción, realización, alegría.

\section{ABSTRACT}

This article describes the validation of the Happiness Scale of Alarcón (2006) in Mexican adolescents, the scale was applied to 405 students from high school, women and men aged 14 to 19 years. The original scale includes correlations item-escala, with coefficients $r$ product moment highly significant $(r=.511 ; p<.001)$ and a Alpha of. 916 . It consists of 27 items of type Likert with five response alternatives. The construct validity of was established by factorial analysis, method of principal components and orthogonal rotation of type Varimax, prior to the calculation of the test of Kaiser-Meyer. Obtained four factors that explain the $50.32 \%$ of total variance; positive sense of the life, satisfaccion with the life, personal realization and happiness of living. In conclusion this scale is appropriate for the measurement of the happiness in this ages.

Keywords: happiness, adolescents, validation, satisfaction, realization.

\footnotetext{
${ }^{*}$ Para correspondencia a cualquiera de los autores: Calle Independencia. No. 603, interior 1, colonia Santa Clara. C. P. 50090. Facultad de Ciencias de la Conducta. Toluca, Estado de México, México. Lorena Toribio Pérez. E-mail. toribio_lore@hotmail.com Tel.7221618321. Norma Ivonne González Arratia López Fuentes. E-mail: nigalf@yahoo.com.mx José Luis Valdez Medina. E-mail: ochocedros@live.com.mx Sergio González Escobar. E-mail: sergioglz4@hotmail.com Hans Oudhof Van Barneveld. E-mail: hansovb@hotmail.com
} 
La felicidad es un tema de la psicología, y ha causado controversia al momento de tratar de medirla (Alarcón, 2006). De acuerdo con Rodríguez (2010), es un estado, pero también un proceso dinámico, generado por la interacción de diferentes condiciones que actúan sobre el individuo provocando respuestas positivas. Estas condiciones pueden ser biológicas (género, salud, malformaciones), psicológicas (rasgos de personalidad, autoestima, valores, creencias afectos) y socioculturales (matrimonio, ingreso económico, familia, marginación, etc.), por lo que en el momento de la medición, los individuos dan diferentes respuestas a las mismas variables. Es así que, el interés por encontrar causas universales que regulan la felicidad ha generado el desarrollo de diferentes escalas para medirla (Alarcón, 2006; Fredrickson, 2009).

Con respecto a la conceptualización, la felicidad es un constructo que ha sido estudiado desde diferentes posturas; desde la filosófica hasta la psicológica. En el primer caso, Sócrates, Aristóteles, Platón y Séneca dieron gran importancia a su estudio, considerándola un bien supremo, un estado en el que no le hace falta nada y la persona se basta a sí misma (Alarcón, 2006).

Posteriormente, otros autores dieron un enfoque psicológico al estudio de la felicidad, entre ellos Maslow, (2006), quien describía a una persona feliz como alguien integrado que lucha pero no contra sí mismo, que es observador, experimentado, inteligente, eficiente y agradable. Utiliza todas sus capacidades en grado e intensidad óptimas. Se siente en funcionamiento pleno, es espontáneo, expresivo y relajado, con mayor confianza y seguridad en sí mismo.

Para definir la felicidad desde el latín se utiliza el término felicitas-atis que significa la satisfacción o posesión de un bien (Alarcón, 2006). De acuerdo con el enfoque hedonista que tiene su origen en filósofos como Aristipo, en el siglo IV a.C., el propósito en la vida era experimentar la máxima cantidad de placer y la felicidad era la totalidad de esos momentos hedónicos (Prados, 2002).

Varios autores (Csickszentmihalyi, 2008; Cuadra \& Florenzano, 2003; Lyubomirsky, 2008; Rodríguez, 1988), coinciden en que la felicidad es un estado mental que las personas pueden llegar a controlar cognoscitivamente, una manera de percibir y de concebirse a sí mismos y al mundo, una experiencia de alegría, satisfacción o bienestar positivo, combinada con la sensación de que la vida tiene sentido.

A pesar de que se ha tratado de definir felicidad, existen divergencias acerca de la objetividad del concepto, ya que es necesario tener claro su significado antes de poder medirla. En algunas investigaciones se ha utilizado el término bienestar subjetivo como sinónimo (Diener \& Biswas, 2008). Según Michalos (1995, citado en Gómez, Villegas, Barrera \& Cruz, 2007), felicidad, bienestar subjetivo y satisfacción tienen un significado común, de manera tal que una teoría de la satisfacción vital debería ser una teoría de la felicidad y ambas se inscribirían en las teorías del bienestar subjetivo.

En lo que se refiere a la medición de felicidad en psicología, esta ha sido mayormente orientada a la identificación de trastornos mentales psicopatológicos tales como ansiedad, depresión, neurosis entre otros. No obstante, también resulta relevante la medición acerca del bienestar y funcionamiento óptimo de los individuos (Cohn \& Fredrickson, 2006; Fredrickson, Coffey, Pek, Cohn \& Finkel, 2008; Seligman, 2011; Vera, 2006).

Específicamente ha resultado compleja la medición de felicidad; las escalas que se han elaborado utilizan constructos como satisfacción con la vida o bienestar subjetivo, para poder medirla. Una de las escalas que se ha desarrollado para tal fin es: la Escala de Satisfacción con la Vida de Diener, Emmons, Larsen y Griffin (1985), la cual está compuesta por cinco preguntas como: "Hasta ahora, he conseguido las cosas que para mí son importantes en la vida”. Las posibilidades de respuesta van de 1 a 7 , donde 1 es completamente en desacuerdo y 7 es completamente de acuerdo. Este instrumento evalúa los aspectos cognitivos del bienestar (Diener, Emmons, Larsen \& Griffin, 1985).

También se publicó en español la Escala de Bienestar Psicológico de Sánchez-Canova (1988). Albuquerque y Trócoli (2009) son autores de una Escala de Bienestar Subjetivo en portugués. El Oxford Happiness Inventory ha sido traducido al hebreo y administrado en Israel (Francis \& Katz, 2000), y ha servido de base para la construcción del Chinese Happiness Inventory, administrado en Taiwan por Lu y Shih (1997) (como cita Alarcón, 2006).

Anguas (2001) realizó un estudio que hace referencia a la validación de una escala que mide bienes- 
tar subjetivo en muestras de mexicanos, el cual refiere que los sentimientos relacionados con este estado fueron preponderantemente positivos, y entre ellos destacan: satisfacción, orgullo, felicidad, y tranquilidad. Asimismo, afirma que es importante que no sólo se adapten instrumentos a la población mexicana, si no que se verifique su validez como confiablidad, a fin de contar con mediciones más precisas de los fenómenos psicológicos.

Con respecto al número de escalas que se utilizan para medir la felicidad, Alarcón (2006) señala que falta mayor investigación al aplicar este tipo de pruebas, dado que pueden existir confusiones en relación con la operacionalización del concepto, por lo que considera necesario que se sigan elaborando instrumentos.

Con el objetivo de medir la felicidad, en algunas investigaciones (Salgado, 2009) se ha utilizado la Escala de Satisfacción con la Vida (Diener, Emmons, Larsen \& Griffin (1985); también se ha usado para medir el bienestar subjetivo (Gómez, Villegas, Barrera \& Cruz, 2007), así como para encontrar su relación con otras variables como la autoestima y ajuste escolar (Anton, Buelga \& Cava, 2007), así como con optimismo (Marrero \& Carballeira, 2010).

Alarcón (2006) menciona que felicidad y bienestar subjetivo se utilizan como sinónimos porque de esta manera se facilita el análisis científico; sin embargo, dice que el término felicidad explica por sí mismo un estado afectivo. Por lo tanto, una escala de felicidad puede contener dimensiones que ayudan a reportar niveles de satisfacción con la vida.

En lo que respecta a la definición este autor (Alarcón, 2006), la felicidad es un sentimiento de satisfacción que vivencia una persona y sólo ella en su vida interior; puede ser duradera, pero a la vez predecible. Un estado afectivo de satisfacción plena que experimenta subjetivamente el individuo en posesión de un bien anhelado. El bien o bienes que generan la felicidad son de naturaleza variada (material, ética, estética, psicológica, religiosa, social, espiritual).

Por otro lado, para evaluarla desarrolló la Escala de felicidad, la cual contempla ítems propios del autor y de otros, como Diener, Emmons, Larsen y Griffin (1985), así como de Hills y Argyle (2002). Esta escala ha sido utilizada en algunas investigaciones (Rodríguez, 2010) para la medición de felicidad.
Una de las variables que se han considerado en el estudio de la felicidad es el sexo, ya que se ha reportado que las mujeres son más proclives a tener mayor felicidad que los hombres (Seligman, 2011). Sin embargo algunos autores (Alarcón, 2006; Argyle, 1990, citado en Gómez et al., 2007; Diener \& Diswas, 2008; Laca, Verdugo \& Guzmán, 2005; Seligman, 2011) están de acuerdo en que el sexo es relevante pero no suficiente para explicar los niveles de felicidad. Por ello resulta pertinente continuar indagando acerca de las diferencias que existen entre hombres y mujeres en los niveles de felicidad.

En México son escasas las investigaciones que hagan referencia a estos niveles en adolescentes, por lo que el objetivo de la presente investigación es obtener los indicadores de validez y confiabilidad de la Escala de Felicidad de Alarcón (2006) para adolescentes, en muestras mexicanas, acerca de su uso en nuestro contexto. Un objetivo adicional es comparar el nivel de felicidad entre hombres y mujeres.

La importancia de validación de la Escala de Felicidad de Alarcón (2006) radica en que se pueden obtener beneficios metodológicos, teóricos y prácticos que permitan ampliar el panorama acerca de felicidad, así como contar con una escala válida para posteriores investigaciones relacionadas con otras variables, tanto de la personalidad; autoestima y optimismo, como sociodemográficas; por ejemplo, sexo, edad, nivel económico.

\section{MÉTODO}

\section{Participantes}

Para el presente estudio se trabajó con una muestra no probabilística de tipo intencional, compuesta por un total de 405 adolescentes: 200 mujeres (49.38\%) y 205 hombres (50.61); todos estudiantes de bachillerato que en el momento de la aplicación del estudio asistían a escuelas preparatorias oficiales de dos municipios del Estado de México: Luvianos y Tejupilco. El rango de edad iba de los 14 a 19 años ( $M=15.82, \mathrm{DE}=1.18)$. Asimismo, reportaron pertenecer a un nivel socioeconómico medio.

\section{Instrumento}

Se aplicó la Escala de Felicidad de Lima (EFL) (Alarcón, 2006), que está compuesta por 27 ítems construidos 
según una escala de tipo Likert de cinco alternativas: totalmente de acuerdo, de acuerdo, ni de acuerdo ni en desacuerdo, en desacuerdo y totalmente en desacuerdo. El extremo positivo tiene un valor de cinco puntos y el extremo negativo un punto. En el cuestionario los ítems están distribuidos aleatoriamente. De acuerdo con el puntaje total se divide en cinco niveles de felicidad: de 27-87: muy baja felicidad; 88-95: baja; 96-110: media; 111-118: alta; 119-135: muy alta.

El autor (Alarcón, 2006) reporta cuatro dimensiones o factores de la felicidad:

Factor 1. Sentido positivo de la vida: los ítems de este factor tal como están formulados, indican profunda depresión, fracaso, intranquilidad, pesimismo y vacío existencial. Las respuestas de rechazo a lo que afirman las oraciones, indican grados de felicidad, que reflejan actitudes y experiencias positivas hacia la vida. En este sentido la felicidad significa estar libre de estados depresivos profundos, tener sentimientos positivos hacia sí mismo y hacia la vida. El factor 1 se compone de 11 ítems, explica el $32.82 \%$ de la varianza total y sus cargas factoriales son elevadas; esta sub-escala posee una alta confiabilidad ( $\alpha=.88$ ).

Factor 2. Satisfacción con la vida: en este factor se expresa satisfacción por lo que se ha alcanzado y la persona cree que está donde debe de estar, o que se encuentra muy cerca de alcanzar el ideal de su vida. Se compone de seis ítems, y explica el $11.22 \%$ de la varianza total. Posee una alta confiabilidad $(\alpha=.79)$.

Factor 3. Realización personal: aquí los reactivos expresan lo que se podría llamar felicidad plena, y no estados temporales del estar feliz. Estos ítems señalan autosuficiencia, autarquía, tranquilidad emocional, placidez; todas son condiciones para conseguir el estado de felicidad completa. El concepto de realización personal guarda correspondencia con la definición de felicidad que supone la orientación del individuo hacia metas que considera valiosas para su vida. Se compone de seis ítems, explica el $10.7 \%$ de la varianza total $(\alpha=.76)$.

Factor 4. Alegría de vivir: la denominación que lleva este factor obedece a que sus ítems señalan lo maravilloso que es vivir, refieren experiencias positivas de la vida y a sentirse generalmente bien. Tiene cuatro ítems y explica el $10.7 \%$ de la varianza total $(\alpha=.72)$ (Alarcón, 2006).
La escala comprende correlaciones ítem-escala, encontrándose coeficientes $r$ producto-momento altamente significativas en todos los ítems $(r=.511 ; p<.001)$ ( $\alpha$ total=.916).

\section{Procedimiento}

Previo a la aplicación se consideró permitente la revisión de la escala. A pesar de que está en español y de que en ambos países se comparte el castellano, fue necesario asegurarse de la comprensión de los ítems. Dicha revisión fue realizada por los autores de la presente investigación y por un jueceo realizado, en el que se obtuvo un acuerdo del $98 \%$ con respecto a que el instrumento es comprensible a muestras mexicanas.

Después la escala fue administrada de manera colectiva en un tiempo de aproximadamente $45 \mathrm{mi}$ nutos, en las aulas de clase de los estudiantes, quienes participaron de manera voluntaria, anónima y confidencial, reportando datos de edad, sexo y promedio académico, y se resolvieron las dudas en el momento de la aplicación.

\section{RESULTADOS}

Para lograr el objetivo del presente estudio se llevaron a cabo análisis descriptivos de correlación y análisis factorial exploratorio para obtener indicadores de validez, así como el cálculo de la confiabilidad a través del Alpha de Cronbach; los datos fueron analizados con el programa spss, versión 16.0. Se codificaron las respuestas de los ítems redactados en forma inversa a la felicidad; 2, 7, 14, 17, 18,19, 20, 22, 23 y 26 . Los puntajes altos indican reacciones positivas hacia la felicidad y los puntajes bajos, baja felicidad.

En primer lugar se procedió a obtener las frecuencias de cada reactivo, así como la media y desviación estándar, con el objetivo de conocer la distribución. En la tabla 1 se observa que los reactivos 12 (es maravilloso vivir) y 19 (me siento un fracasado) resultaron ser altos, mientras que el ítem 27 (creo que no me hace falta nada) puntuó bajo; en el resto de los ítems se aprecia que la media se encuentra por arriba de la media teórica, que es 3 (ver tabla 1). 
Tabla 1. Análisis descriptivo de los reactivos de la Escala de Felicidad de Alarcón (2006)

\begin{tabular}{|c|c|c|c|c|}
\hline Reactivo & Mínimo & Máximo & Media & DE \\
\hline 1. En la mayoría de las cosas mi vida está cerca de mi ideal & 1 & 5 & 3.9 & .8 \\
\hline 2. Siento que mi vida está vacía & 1 & 5 & 4.1 & .9 \\
\hline 3. Las condiciones de mi vida son excelentes & 1 & 5 & 3.7 & .9 \\
\hline 4. Estoy satisfecho con mi vida & 1 & 5 & 4.2 & .8 \\
\hline 5. La vida ha sido buena conmigo & 1 & 5 & 3.8 & 1.0 \\
\hline 6. Me siento satisfecho con lo que soy & 1 & 5 & 4.2 & .8 \\
\hline 7.Pienso que nunca seré feliz & 1 & 5 & 4.2 & 1.0 \\
\hline 8. Hasta ahora he conseguido las cosas que para mí son importantes & 1 & 5 & 3.8 & .9 \\
\hline 9. Si volviese a nacer no cambiaría casi nada en mi vida & 1 & 5 & 3.5 & 1.3 \\
\hline 10. Me siento satisfecho porque estoy donde tengo que estar & 1 & 5 & 4.1 & .9 \\
\hline 11. La mayoría del tiempo me siento feliz & 1 & 5 & 4.1 & .9 \\
\hline 12. Es maravilloso vivir & 1 & 5 & 4.6 & .7 \\
\hline 13. Por lo general me siento bien & 1 & 5 & 4.2 & .8 \\
\hline 14. Me siento inútil & 1 & 5 & 4.2 & 1.0 \\
\hline 15. Soy una persona optimista & 1 & 5 & 3.6 & 1.0 \\
\hline 16. He experimentado la alegría de vivir & 1 & 5 & 4.2 & .8 \\
\hline 17. La vida ha sido injusta conmigo & 1 & 5 & 3.7 & 1.1 \\
\hline 18. Tengo problemas tan hondos que me quitan la tranquilidad & 1 & 5 & 3.5 & 1.2 \\
\hline 19. Me siento un fracasado & 1 & 5 & 4.4 & .8 \\
\hline 20. La felicidad es para algunas personas, no para mí & 1 & 5 & 4.2 & 1.0 \\
\hline 21. Estoy satisfecho con lo que hasta ahora he alcanzado & 1 & 5 & 4.1 & .9 \\
\hline 22. Me siento triste por lo que soy & 1 & 5 & 4.2 & 1.0 \\
\hline 23. Para mí la vida es una cadena de sufrimientos & 1 & 5 & 4.1 & 1.1 \\
\hline 24. Me considero una persona realizada & 1 & 5 & 3.7 & .9 \\
\hline 25. Mi vida transcurre plácidamente & 1 & 5 & 3.6 & 1.0 \\
\hline 26. Todavía no he encontrado sentido a mi existencia & 1 & 5 & 3.7 & 1.0 \\
\hline 27. Creo que no me hace falta nada & 1 & 5 & 3.3 & 1.1 \\
\hline
\end{tabular}


Tabla 2. Estructura factorial de la Escala de Felicidad

Matriz de factores extraídos por rotación Varimax cargas factoriales de los ítems

\begin{tabular}{|c|c|c|c|c|}
\hline Ítems & F1 & F2 & F3 & F4 \\
\hline 23. Para mí, la vida es una cadena de sufrimientos & 0.77 & & & \\
\hline 20. La felicidad es para algunas personas, no para mí & 0.75 & & & \\
\hline 19. Me siento un fracasado & 0.73 & & & \\
\hline 22. Me siento triste por lo que soy & 0.69 & & & \\
\hline 18. Tengo problemas tan hondos que me quitan la felicidad & 0.67 & & & \\
\hline 7. Pienso que nunca seré feliz & 0.64 & & & \\
\hline 26. Todavía no he encontrado sentido a mi existencia & 0.6 & & & \\
\hline 17. La vida ha sido injusta conmigo & 0.55 & & & \\
\hline 2. Siento que mi vida está vacía & 0.53 & & & \\
\hline 14. Me siento inútil & 0.46 & & & \\
\hline
\end{tabular}

1. En la mayoría de las cosas mi vida está cerca de mi ideal $\quad 0.71$

$\begin{array}{ll}\text { 3. Las condiciones de mi vida son excelentes } & 0.65\end{array}$

$\begin{array}{ll}\text { 5. La vida ha sido buena conmigo } & 0.59\end{array}$

$\begin{array}{ll}\text { 4. Estoy satisfecho con mi vida } & 0.59\end{array}$

$\begin{array}{ll}\text { 6. Me siento satisfecho con lo que soy } & 0.52\end{array}$

10. Me siento satisfecho porque estoy donde tengo que estar 0.47

25. Mi vida transcurre plácidamente 0.72

27. Creo que no me hace falta nada $\quad 0.65$

24. Me considero una persona realizada $\quad 0.55$

21. Estoy satisfecho con lo que hasta ahora he alcanzado $\quad 0.44$

9. Si volviese a nacer, no cambiaría casi nada en mi vida 0.402

13. Por lo general me siento bien 0.43

15. Soy una persona optimista 0.66

16. He experimentado la alegría de vivir 0.64

$\begin{array}{ll}\text { 12. Es maravilloso vivir } & 0.57\end{array}$

11. La mayoría del tiempo me siento feliz 0.46

\begin{tabular}{|c|c|c|c|c|}
\hline Varianza por factor & 18.123 & 11.229 & 10.702 & 10.27 \\
\hline Varianza total & & & & 50.324 \\
\hline Alfa de Cronbach por factor & 0.913 & 0.913 & 0.915 & 0.914 \\
\hline Alfa de Cronbach total & & & & 0.917 \\
\hline
\end{tabular}


En seguida se procedió a obtener indicadores de validez, por lo que se realizó un análisis factorial exploratorio para observar la estructura subyacente de la Escala de Felicidad. Previo al cálculo de la prueba de Kaiser-Meyer (.933, p=.001), los resultados fueron satisfactorios, por lo que se procedió a correr el factorial con un criterio de peso factorial de .40, con el método de componentes principales y rotación ortogonal de tipo Varimax, en donde se obtuvieron cuatro factores, que de acuerdo con su claridad conceptual y punto de quiebre de la varianza explican el $50.32 \%$. Los factores son: 1 . sentido positivo de la vida, 2. satisfacción con la vida, 3. realización personal, y 4. alegría de vivir. Se obtuvo un $\alpha=.917$. De acuerdo con los resultados, se obtuvieron las cuatro dimensiones de la escala original con los mismos reactivos, eliminando únicamente el reactivo número 8 (ver tabla 2 ).

Con el objetivo de observar si existían diferencias en el nivel de felicidad se aplicó la $t$ de Student, según la variable atributiva sexo, con lo cual se puede ver que no existen diferencias estadísticamente significativas entre hombres y mujeres en los niveles obtenidos por dimensión; con respecto a la media, las mujeres puntúan ligeramente más alto en las dimensiones, sentido positivo de la vida y alegría de vivir, y los hombres en satisfacción con la vida y realización personal (ver tabla 3).

Asimismo, se realizó un análisis de correlación entre las dimensiones de la Escala de Felicidad, donde se puede observar que existen correlaciones positivas altas y estadísticamente significativas entre las dimensiones. Con respecto a la dimensión, sentido positivo de la vida y satisfacción con la vida $\left(\mathrm{r}=.538^{\star *}, \mathrm{p}=.01\right)$, a realización personal $\left(\mathrm{r}=.466^{\star *}, \mathrm{p}=.01\right)$ y a alegría de vi$\operatorname{vir}\left(\mathrm{r}=.540^{\star *}, \mathrm{p}=.01\right)$. La dimensión satisfacción con la vida y realización personal $\left(\mathrm{r}=.649^{\star *}, \mathrm{p}=.01\right)$, así como con alegría de vivir $\left(r=.648^{\star *}, \mathrm{p}=.01\right)$. Finalmente, realización personal se asocia positivamente con alegría de vivir $\left(\mathrm{r}=.585^{\star \star}, \mathrm{p}=0.1\right)$ (ver tabla 4$)$.

Tabla 3. $t$ de Student de Felicidad por factores según sexo

\begin{tabular}{|c|c|c|c|c|c|c|}
\hline Factores & Hombres & $\mathrm{n}=\mathbf{2 0 5}$ & Mujeres & $n=200$ & $\mathrm{p}$ & $t$ \\
\hline & Media & $\begin{array}{l}\text { Desviación } \\
\text { Estándar }\end{array}$ & Media & $\begin{array}{c}\text { Desviación } \\
\text { Estándar }\end{array}$ & & \\
\hline 1. Sentido positivo de la vida & 4.07 & .73 & 4.10 & .75 & .266 & 1.240 \\
\hline 2. Satisfacción con la vida & 4.05 & .64 & 4.00 & .70 & .190 & 1.723 \\
\hline 3. Realización personal & 3.68 & .73 & 3.67 & .69 & .816 & .055 \\
\hline 4. Alegría de vivir & 4.19 & .62 & 4.21 & .60 & .276 & 1.192 \\
\hline
\end{tabular}

Tabla 4. Análisis de correlación entre las dimensiones de Felicidad

\begin{tabular}{|c|c|c|c|c|}
\hline Dimensiones & 1 & 2 & 3 & 4 \\
\hline 1. Sentido positivo de la vida & & $.538 * *$ & $.466^{* *}$ & $.540 * *$ \\
\hline 2. Satisfacción con la vida & & & $.649 * *$ & $.648 * *$ \\
\hline 3. Realización personal & & & & $.585^{* *}$ \\
\hline
\end{tabular}

4. Alegría de vivir

** $\mathrm{p}=.01 \quad * \mathrm{p}=.05$

Sentido positivo de la vida. 2. Satisfacción con la vida. 3. Realización personal. 4. Alegría de vivir. 


\section{DISCUSIÓN}

La felicidad ha sido un concepto muy estudiado; son cada vez más las contribuciones que se hacen al respecto. En la presente investigación se llegó a hallazgos interesantes donde se observan resultados que coinciden con las aportaciones de otros autores (Alarcón, 2006; Seligman, 2011).

Con respecto a la validez de esta escala, el estudio psicométrico comprendió un análisis factorial exploratorio de componentes principales y rotación Varimax, en el cual se obtuvo una estructura con las mismas dimensiones y número de reactivos, que la Escala de Felicidad de Alarcón (2006); sólo se eliminó el ítem 8. Se conservaron 26 de los 27 reactivos, así como el orden de las dimensiones de Alarcón (2006), las cuales son: 1. sentido positivo de la vida, 2. satisfacción con la vida, 3. realización personal y 4. alegría de vivir. También se obtuvieron correlaciones entre las dimensiones estadísticamente significativas. Con esto se comprueba que cada ítem corresponde a la dimensión correcta y mide lo que quiere medir. Se obtuvo un a total $=.917$.

En este sentido se puede pensar que el contenido de los ítems del instrumento no tiene restricciones culturales, ya que además de la revisión previa del mismo por parte de los autores, los hallazgos psicométricos permiten considerarlo útil para una población adolescente diferente para la que originalmente fue elaborado el instrumento (Anastasi \& Urbina, 1998).

En lo que respecta a los niveles de felicidad, se obtuvieron resultados semejantes a los del autor con muestras peruanas, ya que un 36\% de la muestra de esta investigación se encuentra en el nivel medio de felicidad. Estos resultados coinciden con Csikszentmihalyi y Selega (1998), quienes en estudios anteriores encontraron que los adolescentes son capaces de obtener estados no tan sólo de salud óptima si no de ser felices, ya que se sienten satisfechos consigo mismos, con lo que tienen y con las metas que han alcanzado. Entre las explicaciones acerca de la felicidad autorreportada, la investigación refiere que es multifactorial, e incluye tópicos como el nivel de estudios, clima, raza, nivel socioeconómico y lugar de origen (Alarcón, 2006; Diener \& Biswas, 2008; Laca et al., 2005; Seligman, 2011). Si bien estos indicadores no se consideraron en el presente estudio, sería recomendable indagarlos en las posteriores investigaciones, a fin de precisar la naturaleza de la felicidad con la juventud.

Como uno de los objetivos fue identificar si existen diferencias de felicidad por sexo, al realizar el análisis con la $t$ de Student, no se encontraron diferencias estadísticamente significativas entre hombres y mujeres; lo cual quiere decir que independientemente del género, ambos sienten mayor satisfacción por lo que han alcanzado, creen estar donde deben estar y se encuentran muy cerca de alcanzar el ideal de su vida; asimismo, se consideran autosuficientes, tienen tranquilidad emocional, se orientan a metas que consideran valiosas para su vida, experimentan sentimientos positivos hacia sí mismos y hacia la vida, sienten que es maravilloso vivir y refieren experiencias positivas de su vida (Alarcón, 2006; Csikszentmihalyi, 2008; Maslow, 2006).

Entre las explicaciones de estas no diferencias, refiere Seligman (2011), que son más importantes otro tipo de factores como el tipo de familia, tener o no una pareja, mantener buen estado de salud, juventud y religión para influir en la felicidad.

Tener sentido de vida significa que los adolescentes se sienten bien consigo mismos, son conscientes de sus fortalezas y capacidades. De acuerdo con Csikszentmihalyi y Selega (1998), cuando los jóvenes viven experiencias que los hacen sentirse más auténticos, se aceptan a sí mismos, tienen características marcadas que les permiten sentirse plenos y capaces de lograr sus metas. Se puede decir que los adolescentes son personas sanas, que pueden potenciar las fortalezas humanas, y por lo tanto son capaces de afrontar las adversidades y ser mejores seres humanos (Gable \& Haidt, 2005; González, 2004; Maslow, 2006; Vázquez, 2006).

Los resultados con respecto a la validez y confiabilidad indican que la Escala de Felicidad aplicada en adolescentes mexicanos cuenta con las propiedades psicométricas adecuadas para considerarse como una medida válida y confiable (Alarcón, 2006). Esto quiere decir que se puede aplicar en futuras investigaciones con la finalidad de observar si existen relaciones con otras variables de tipo sociodemográficas; sexo, edad, nivel socioeconómico, así como con variables de personalidad como autoestima y optimismo.

Con base en estos hallazgos se considera que si se utilizan escalas desarrolladas en otros contextos, éstas 
deben contar con cualidades psicométricas que permitan avanzar en la medición de diversos constructos. En este caso, la escala cuenta con características importantes que la hacen adecuada y relevante a nuestra

\section{REFERENCIAS}

Anastasi, A. \& Urbina, S. (1998). Tests psicológicos. (Séptima edición). México: Prentice Hall.

Alarcón, R. (2006). Desarrollo de una escala factorial para medir la felicidad. Revista Interamericana de Psicología, 40(1), 96-106.

Anton, M., Buelga, M. \& Cava, S. (2007). La satisfacción con la vida en la adolescencia y su relación con la autoestima en el ajuste escolar. Anuario de Psicología, 38(2), 293-303.

Cohn, M. \& Fredrikson, B. (2006). Beyond the moment beyond the self: shared Ground Between. Selective investment theory and the Broaden-andbuild. Theory of Positive Emotions. Universidad de Michigan. Psychological Inquiry, 17(1), 39-44.

Cuadra, H. \& Florenzano, R. (2003). La psicología positiva: un cambio a nuestro enfoque patológico clásico. Liberabit. Revista de Psicología de Lima, Perú, 10, 82-88.

Csikszentmihalyi, M. \& Selega, I. (1998). "Experiencia Óptima: estudios psicológicos del flujo en la conciencia”. Bilbao: Descleé De Brouer.

Csikszentmihalyi, M. (2008). El yo evolutivo. Una psicología para un mundo globalizado. Barcelona: Kairos.

Diener, E., Emmons, R., Larsen, R. J. \& Griffin, S. (1985). The satisfaction with life scale. Journal of Personalitiy Assessment, 49, 71-75.

Diener, E., \& Diswas, R. (2008). Happiness. Unlocking the Mysteries of psychological wealth. E. U. A.: Blackwell Publishing.

Fredrickson, B. (2009). Positivity. Nueva York, Estados Unidos: Random House Inc.

Fredrickson, B. L., Coffey, K., Pek, J., Cohn, M. \& Finkel, S. (2008). Open Hearts Build Lives: Positive Emotions, Induced Through Loving-Kidness Meditation, Build Consequential Personal Resources. A. P. A. Journal of Personality and Social Psichology, 95(5). 1045-1062.

Gable, S. \& Haid, J. (2005). What (and why) Is Positive Psychology? Review of General Psychology, 9(2), 103-110. cultura. Sin embargo, resulta indispensable continuar su uso en una muestra ampliada a fin de seguir verificando su utilidad en muestras mexicanas.

González, C. (2004). La psicología positiva: un cambio en nuestro enfoque patológico. Revista de Psicología, 10, 82-88.

Gómez, V., Villegas, C. Barrera, F. \& Cruz, J. (2007). Factores predictores de bienestar subjetivo en una muestra colombiana. Revista Latinoamericana de Psicología, 39(2), 311-125.

Laca, F., Verdugo, J. \& Guzmán, M. (2005). Satisfacción con la vida de algunos colectivos mexicanos: una discusión sobre la psicología del bienestar subjetivo. Enseñanza en Investigación en Psicología, 10(2), 325-336.

Lyubomirsky, S. (2008). La ciencia de la felicidad. Un método para conseguir el bienestar.

España: Urano.

Maslow, A. (2006). La amplitud potencial de la naturaleza humana. (2da. Ed.). México: Trillas.

Prados, F. (2002). Disfrute y bienestar subjetivo. Un estudio psicométrico de la gaudibilidad. (Tesis doctoral no publicada). Universidad Autónoma de Barcelona: España.

Rodríguez, C. (2010). Variables psicológicas asociadas con la felicidad en centros peri-urbanos y urbanos marginales de Lima. UCV-Scientia, 2(1), 61-68.

Rodríguez, J. M. (1988). La felicidad. Madrid, España: Temas de hoy.

Salgado, A. (2009). Felicidad, resiliencia y optimismo en estudiantes de colegios nacionales de la ciudad de Lima. Liberabit. Revista de Psicología, Lima, 15 (2), 133-141.

Seligman, M. (2011). La auténtica felicidad. España: Byblos.

Vázquez, C. (2006). La psicología positiva en perspectiva. Papeles del Psicólogo, 27 (1), 1-2.

Vera, B. (2006). Psicología positiva, una nueva forma de entender la psicología. Papeles del Psicólogo, 27(1), 3-8.

Fecha de recepción: Noviembre 2011 Fecha de publicación: Junio 2012 